\title{
A Numerical Model for Multiple Detector Energy Dispersive X-ray Spectroscopy in the Transmission Electron Microscope
}

\author{
W. Xu ${ }^{\mathrm{a}}$, J. H. Dycus ${ }^{\mathrm{a}}$, X. Sang ${ }^{\mathrm{a}}$, J. M. LeBeau ${ }^{\mathrm{a}, *}$ \\ ${ }^{a}$ Department of Materials Science and Engineering, North Carolina State University, Raleigh, NC 27695, USA.
}

\begin{abstract}
Here we report a numerical approach to model a four quadrant energy dispersive x-ray spectrometer in the transmission electron microscope. The model includes: detector geometries, specimen position and absorption, shadowing by the holder, and filtering by the Be carrier. We show that this comprehensive model accurately predicts absolute counts and intensity ratios as a function of specimen tilt and position. We directly compare the model to experimental results acquired with a FEI Super-X EDS four quadrant detector. The contribution from each detector to the sum is investigated. The program and source code can be downloaded from https://github.com/subangstrom/superAngle.
\end{abstract}

Keywords: Energy dispersive X-ray spectroscopy (EDS), Super-X, detector collection angle, X-ray absorption correction, shadowing, spurious X-rays

\section{Introduction}

Energy Dispersive X-ray Spectroscopy (EDS) is widely used for qualitative and quantitative elemental composition analysis at the nanometer scale [1]. Recent developments have focused on improving the X-ray signal collection efficiency by either adding more detectors or using a larger collection area [2]. For example, the FEI Super-X system incorporates four windowless $30 \mathrm{~mm}^{2}$ SSD detectors that are placed symmetrically around the optic axis. The total collection angle of such systems is 0.7 or $0.9 \mathrm{sr}$ depending on the pole piece geometry $[3,4]$. Furthermore, the use of windowless detectors in this configuration further increases the collection efficiency for light elements [5, 6].

Advances in EDS detector technology, paired with high brightness of field emission guns and aberrationcorrection, have enabled routine atomic-resolution EDS elemental mapping. With this capability, attention has turned to quantifying elemental composition on an atom column by atom column basis [7-13]. Beyond accounting for the complexities of electron channeling, quantitative atomic resolution elemental mapping also requires knowledge of detector collection solid-angle [13]. When the sample is titled however, e.g. to

\footnotetext{
* Corresponding author

Email address: jmlebeau@ncsu.edu (J. M. LeBeau) 
zone axis condition necessary for atomic resolution, the full collection solid angle can be reduced if the specimen holder shadows the detector $[14,15]$.

In the Super-X configuration, at least one of the four detectors will be inevitably blocked or shadowed as the sample holder tilt increases. Furthermore, specimen shift can play a role in determining which X-rays are collected. Beyond the holder and sample, the Be specimen carrier can also absorb a significant fraction of the X-ray signal, especially for light elements. Rather than an absolute number then, the effective collection solid angle will change as the sample is tilted and/or shifted, which cannot be accounted for from calibration alone. As a result, absolute EDS quantification at the atomic scale becomes even more challenging. Instead, a sufficiently complex theoretical model to predict the variation of effective collection angle or absorption correction factors with the specimen tilt and shift would provide critical insights and information. Recently, Yang et al[16] have provided analytical solutions for absorption correction in the Super-X configuration while Yeoh et al. considered the effects of holder shadowing in EDS tomography [15]. Slater et al. also reported optical measurements of a tomography holder to better integrate holder geometry in EDS tomography quantification [17]. A more complete model, however, is still lacking.

In this work, a comprehensive numerical model that includes the precise geometry of the specimen holder to determine the combined effects of specimen absorption, Be holder absorption, and holder shadowing is developed. We find that when tilting the specimen, the geometric variance between the specimen and each detector introduces important contributions that should be taken into account, particularly when tilting beyond even $10^{\circ}$. Furthermore, using $\mathrm{Ni}_{3} \mathrm{Al}$ as a test case, we validate the model by directly comparing total absolute counts and intensity ratios from experiment with those calculated with the model. Through the model, we provide fundamental insights into the complexities of how X-rays reach each detector as a function of tilt and position. The results provide important input to achieve accurate quantification as detector geometries become increasingly complex.

\section{Materials \& Methods}

Samples of $\mathrm{Ni}_{3} \mathrm{Al}$ were prepared either by wedge polishing or focused ion beam (FIB). The wedge polished sample was first mechanically thinned with an Allied Mulitprep system using diamond lapping films, and subsequently ion-milled to electron transparency with a Fischione 1050 ion mill. The FIB sample was prepared using an FEI Quanta 3D FEG dual-beam instrument, which was was directly cut from a bulk sample and attached in the middle of ' $\mathrm{M}$ ' shaped PELCO ${ }^{\circledR}$ Mo grid. The sample was then further thinned using $30 \mathrm{kV} \mathrm{Ga}^{+}$ions that was then stepped down to $5 \mathrm{kV}$ and $2 \mathrm{kV}$ for final polishing.

A probe-corrected FEI Titan G2 microscope operated at $200 \mathrm{kV}$ and equipped with a four-quadrant FEI Super-X detector was used throughout. A FEI low background double tilt specimen holder (LB DT HiVis specimen holder FP6595/20) was used. EDS spectra were acquired along X- and Y-tilt axes using an area of 
about $200 \times 200 \mathrm{~nm}^{2}$ to reduce beam damage and carbon contamination. At each specimen tilt, the signal on each of the four Super-X detector segments was acquired individually. The probe current, measured using a calibrated CCD, was approximately 121 and $95 \mathrm{pA}$ for the wedge-polished and FIB samples respectively. The CCD was calibrated using a picoammeter, with a precision of about $0.1 \mathrm{pA}$, connected to the EELS drift tube. The EDS acquisition rate was in the range of 0.1-1.7 kcps per detector depending on the tilt. For each spectrum, the live time was 69 and 135 s for FIB and wedge-polished samples respectively. Note that, the live time $\tau$ was different from the total experimental acquisition time, influenced by the dwell time for each pixel. Strong electron channeling conditions were avoided. Furthermore, the acquisition area was kept approximately constant. For the wedge-polished specimen, the acquisition position was at approximately $-0.11 \mathrm{~mm},-0.12 \mathrm{~mm}$ and $0.29 \mathrm{~mm}$ along $\mathrm{x}, \mathrm{y}$ and $\mathrm{z}$ directions relative to the optic axis. The FIB sample was shifted $0.37 \mathrm{~mm}, 0.02 \mathrm{~mm}$ and $0.22 \mathrm{~mm}$ along $\mathrm{x}$, $\mathrm{y}$ and $\mathrm{z}$.

The tilt series data were acquired at approximately the same thickness, as monitored by electron energy loss spectroscopy (EELS) with a collection semi-angle of 39 mrad. Compared to using a constant location, this strategy largely reduced the possible position variation or uncertainty during the $\mathrm{X} / \mathrm{Y}$ tilt experiment. The sample thickness was kept at a constant value of 1.0 inelastic mean free path for the wedge-polished sample, and 1.5 inelastic mean free paths for the FIB sample. This corresponds to thicknesses of 84 and 126 $\mathrm{nm}$ for the wedge-polished and FIB samples, respectively, as estimated from the Malis model with error of approximately $\pm 10 \%[18,19]$

\section{Description of the numerical model}

Figure 1a shows a geometric overview of a FEI Super-X detector, with the specimen placed at the origin. Four detectors are symmetrically placed about the sample with a polar angle, $\theta=18^{\circ}$, and azimuth angles, $\varphi_{D}$, of $135^{\circ}, 45^{\circ}, 315^{\circ}, 225^{\circ}$, respectively. To discretize the detector, differential elements are given by $d \theta$ and $d \varphi$, as in Figure 1b. A factor of $\sin \theta_{i}$ is included to normalize the intensity of each X-ray. Note that the index $i$ indicates a discrete X-ray detector element, or equivalently a X-ray trajectory from the sample. The solid angle for each differential detector element is then:

$$
d \Omega=d \theta d \varphi \sin \theta_{i}
$$

After discretization, only those X-rays that pass into the detector area are considered. Based on the central point, $P_{i}$, that each X-ray passes through a detector, we define the plane normal of that detector to be $\mathbf{n}=\left[1, \theta_{D}+\delta_{D}, \varphi_{D}\right]$ where $\delta_{D}$ is the angle that the detector could be tilted along the $\mathbf{O P} \times \mathbf{P Q}$ direction,

$$
\left(\mathbf{O P}_{i}-\mathbf{O P}_{0}\right) \cdot \mathbf{n}=0
$$



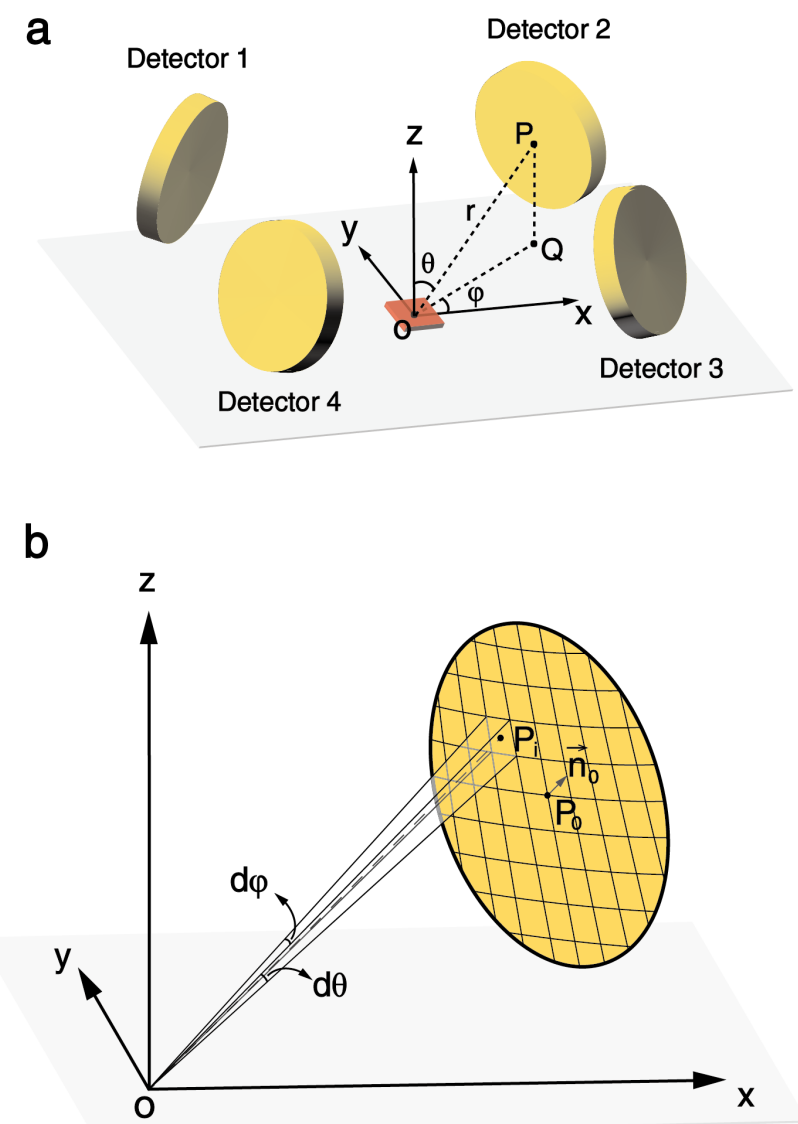

Figure 1: (a) Super-X detector quadrants placed symmetrically about the sample. The specimen is placed at the origin point, $\mathrm{O}$, at distance $r$ from the detectors. $P$ and $Q$ define points within each detector plane and projected onto the specimen plane, respectively. (b) X-rays generated within the specimen are discretized onto the detector surface, defined by surface normal $\mathbf{n}_{0}$, within differential solid angle elements centered about points $P_{i}$.

By solving Equation 2, we obtain a large set of intercept coordinates $\left(\mathrm{P}_{i}\right)$ from the X-rays that pass through the detector plane. This process is then repeated for each of the four different detectors. By considering only those intercepts with $\left|\mathbf{P}_{i} \mathbf{P}_{0}\right|$ less than the detector radius, the complete set of the X-rays, $\mathbf{O} \mathbf{P}_{i}$, that can be collected by the detectors is determined. For convergence, $\mathrm{d} \theta$ and $\mathrm{d} \varphi$ intervals of $0.2^{\circ}$ are sufficient to fully describe the detectors. Note that this numerical approach can be used to accommodate any arbitrary number of detectors or detector shape by modifying the above criteria.

As the nominal total solid angle is $0.7 \mathrm{sr}$ for the Super-X system, the distance from the specimen to the center of the detector $\left|\mathbf{O P}_{0}\right|$ is approximately $12.0 \mathrm{~mm}$. Here, we consider only the collimated area of the detector $\left(26.4 \mathrm{~mm}^{2}\right)$, i.e. active detector radius of $2.9 \mathrm{~mm}[20]$, and a detector elevation angle of $18^{\circ}$ (or $\theta_{D}=72^{\circ}$ ). $\delta_{D}$ is assumed to be zero due to the lack of manufacture information, which proves to be reasonable as the results are found to be insensitive to its variation. 
It should be noted that the model incorporates knowledge of specimen to predict the detector-collection angle, including specimen thickness, depth, coordinates of the specimen with the pole-piece gap, specimen grid geometry (if it happens to block the X-ray signal) and the inclination angle between specimen and the primary axis of the holder, i.e. specimen geometry. Other factors related to the microscope such as the geometry of detector, holder frame, specimen carrier, Softlock securing clip are directly incorporated without additional inputs. Furthermore, we distinguish between selective filtering effect (which absorbs a certain ratio of low energy X-rays) from shadowing effect (which blocks X-rays).

Before delving into further details, we highlight a variety of factors that will also influence which of the X-rays leaving the sample can reach one of the four quadrants. To do so, we introduce an effective detector solid-angle, $\Omega_{\text {eff }}$ that is determined by summing over all discrete, $i$, detector positions and sample thicknesses, $t$.

$$
\Omega_{\mathrm{eff}}=\frac{1}{N} \sum_{t} \sum_{i} B_{i, \text { frame }} B_{i, \text { clip }} B_{i, \text { grid }} A_{i, \text { spec }}(t) A_{i, \text { carr }} d \theta d \varphi \sin \theta_{i}
$$

The $A_{i, x}$ factors include the absorption by the specimen, $A_{i, \mathrm{spec}}$, and absorption by the sample carrier,

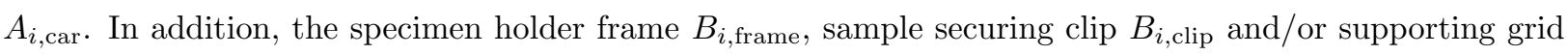
$B_{i \text {,grid }}$ may absorb nearly $100 \%$, i.e. block, of the X-rays. Each contribution is determined according to the model presented below.

The above model can be incorporated to predict the absolute X-ray intensity, which is proportional to the solid angle and composition according to [21],

$$
I_{i}=N_{V} I_{e} \tau \rho t \frac{Q_{i} \omega_{i} a_{i} \epsilon_{i}}{e M} \frac{\Omega}{4 \pi} C_{i}
$$

where $\mathrm{N}_{v}$ is Avogadro's number, $I_{e}$ is the incident probe current, $e$ is the elementary charge, $\tau$ is the live time of signal acquisition, $\rho$ is the specimen density, $t$ is the specimen thickness, $Q_{i}$ is the ionization cross-section, $\omega_{i}$ is fluorescence yield, $a_{i}$ is the relative transition probability, $\epsilon_{i}$ is the detector efficiency, $M_{i}$ is atomic mass, $C_{i}$ (or $C_{B}$ ) is the elemental concentration in the specimen. For $\mathrm{Ni}_{3} \mathrm{Al}$ specimen, $Q_{\mathrm{Al}-\mathrm{K}}$ and $Q_{\mathrm{Ni}-\mathrm{K}}$ are $2264 \operatorname{barn}[22]$ and $255 \operatorname{barn}[23], \omega_{\mathrm{Al}-\mathrm{K}}$ and $\omega_{\mathrm{Ni}-\mathrm{K}}$ are 0.0357[24] and 0.414[23], $a_{\mathrm{Al}-\mathrm{K}}$ and $a_{\mathrm{Ni}-\mathrm{K}}$ are 1 , considering all K-shell x-rays, $\epsilon_{\mathrm{Al}-\mathrm{K}}$ and $\epsilon_{\mathrm{Ni}-\mathrm{K}}$ are 0.982 and $0.980[4]$, respectively. The calculated effective solid angle in the model can be directly integrated into composition analysis of experimental measurements of X-ray intensities for different elements. Specifically, the predicted $\Omega_{\mathrm{eff}}$ and can then be incorporated into the Cliff-Lorimer equation for different elements [25],

$$
\frac{C_{A}}{C_{B}}=k_{A B} \frac{\Omega_{e f f, B}}{\Omega_{e f f, A}} \frac{I_{A}}{I_{B}}
$$

where $\Omega_{e f f, B} / \Omega_{e f f, A}$ is then a correction factor to the ideal k-factor $k_{A B}[1]$. 


\subsection{Shadowing by the specimen holder}

As detailed above, incorporation of the detector geometry alone is insufficient to fully account for the absorption and blocking effects. For example, consider the FEI sample holder shown schematically in Figure 2. The holder is constructed of a brass holder frame, a Be specimen carrier, and a Softlock securing clip. The machined parts are illustrated via different colours. The specimen support grid, which is usually $\mathrm{Mo}$ or $\mathrm{Cu}$, is also included. When the specimen is tilted or shifted relative to the detectors, X-rays generated by the specimen may be blocked by the specimen holder depending upon its geometry and material. In conventional single detector EDS, holder shadowing can be avoided by tilting the specimen toward the detector. This approach fails, however, for the Super-X configuration. A favourable specimen tilt toward one detector will cause unfavourable shadowing effects for the other detectors (see Supplement Movie 1). Absolute quantification in Super-X configuration can thus require that this contribution be taken into account.

a

Top view Detector 2

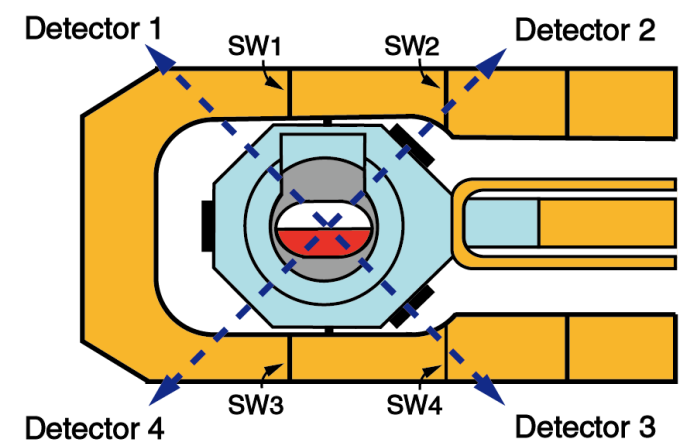

b

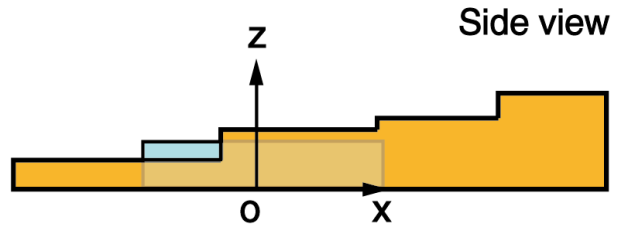

Specimen

$\square$ Specimen grid

Specimen carrier $(\mathrm{Be})$

Softlock clip (Mo/Cu)

Holder frame

Figure 2: (a) Illustration of the specimen holder, including the specimen (red), holder frame (yellow), specimen carrier (blue), Softlock securing clip (black), and specimen grid (grey). (b) Side-view of the specimen holder, highlighting the stepwise holder frame, with its left portion shorter than the right.

Among the X-rays generated by the sample, those exiting toward the inner wall of the holder frame are blocked, introducing shadowing. The inner wall shows axial symmetry along its horizontal center line, but shows no axial symmetry along the vertical center line co-linear with the specimen center. This geometric feature introduces a shorter distance between the specimen to the wall (OD) in Figure $3 a$ than that in 
Figure 3b. In addition, the holder frame has stepwise geometry on its side view as shown in Figure 2b. Its left portion is shorter than the right portion, which reduces the degree of X-ray shadowing along the path to detectors 1 and 4 compared with that to detectors 2 and 3 . In addition to the inner wall, the sidewalls on the frame step can also block the X-ray (marked as SW1-SW4 in Figure 2), making the consideration of holder shadowing more complicated.

a

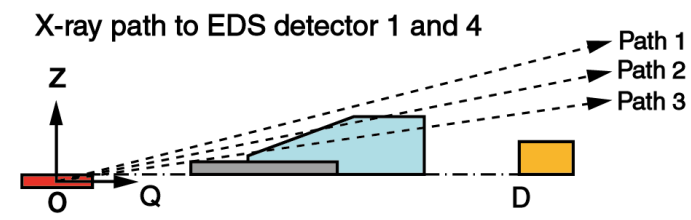

b

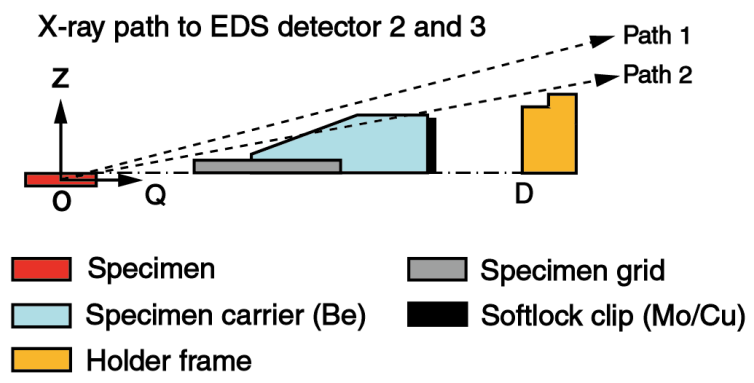

Figure 3: (a) X-ray paths from the specimen to detectors 1 and 4. The holder frame does not block the X-rays in this orientation. (b) X-ray paths from the specimen to detectors 2 and 3. Under the same condition as (a), the holder frame and Softlock securing clip partially block X-rays, causing shadowing.

The holder frame can only rotate about the x-axis (equivalent to the FEI $\alpha$ tilt control), represented by the rotational matrix $\mathbf{R}_{x}$. The model also considers the specimen movement in the TEM stage, represented by a translational vector $T=\left[T_{x}, T_{y},-T_{z}\right] . T_{x}$ and $T_{y}$ are directly obtained from position reading in $\mathrm{x}$ and $\mathrm{y}$ coordinate in the microscope. $T_{z}$ is the depth difference between the specimen and top surface of the holder carrier. $T_{z}$ can be measured experimentally by comparing the relative microscope z-coordinate between a test specimen and a FIB-prepared specimen attached close the top surface of the FIB grid. The latter is placed in the ideal specimen depth of about $0.22 \mathrm{~mm}$ according to the geometric information in the holder manual provided by the manufacture.

As we have discretized the X-rays that can reach the detectors, we need to further reject X-rays that will be blocked by shadowing. To do so, the geometry of the holder is first measured from optical microscopy images. A complete set of coordinates $\mathbf{M}$, which represents the upper boundary of the inner sidewall of the holder frame is obtained with intervals as small as $0.1 \mathrm{~mm}$. For each X-ray, a coordinate $M_{j}\left(x_{j}, y_{j}, z_{j}\right)$ from the set $\mathbf{M}$ is obtained by searching the minimum angle between the X-ray path and $\mathbf{O M}_{\mathbf{j}}$, which represents the shortest distance from holder boundary to the X-ray. The inner sidewall that passes the 

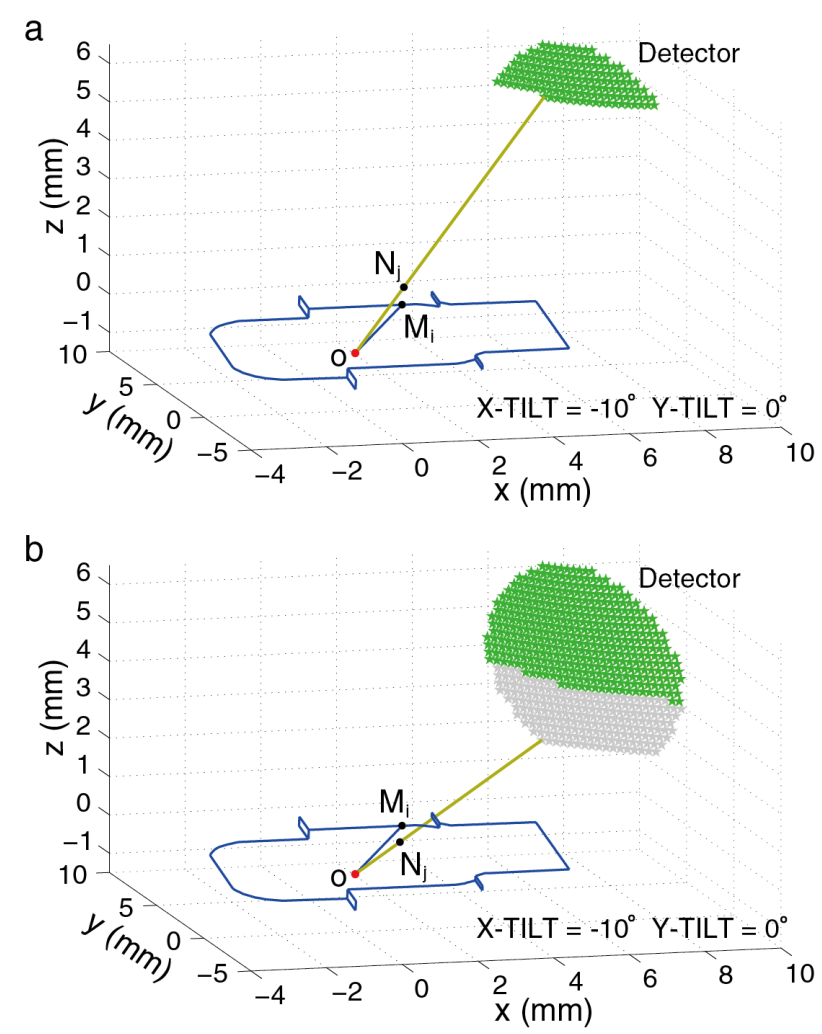

Figure 4: Two frames from Supplement Movie 2 showing X-ray (a) acceptance and (b) rejection at -10 degrees of X-tilt. Non-shadowed detector positions are marked green, while those rejected X-rays are marked as gray. The blue lines indicate the upper boundary of the specimen holder sidewalls.

holder frame boundary $M_{j}\left(x_{j}, y_{j}, z_{j}\right)$ is then defined by its plane normal $n_{j}$. The holder tilt about x-axis is also considered, representing as a rotational matrix $\mathbf{R}$. We then solve the following equation to obtain the intercept point $N_{i}\left(x_{i}, y_{i}, z_{i}\right)$ of this X-ray on the vertical plane of the inner sidewall.

$$
\left(\mathbf{n}_{j} \mathbf{R}\right) \cdot\left[\left(\mathbf{O M}_{j}-\mathbf{T}\right) \mathbf{R}-\mathbf{O N}_{i}\right]=0
$$

If $N_{i}\left(z_{i}\right)$ is greater than $M_{j}\left(z_{j}\right)$, this X-ray will be accepted; otherwise, it will be rejected. Note that for X-rays traveling close to the frame step corner, additional comparison between $N_{i}\left(x_{i}\right)$ and $M_{j}\left(x_{j}\right)$ is needed. We also note that this algorithm reduces the dimension of holder frame shadowing model from 3D to 2D, which largely improves the computational efficiency.

The holder sidewalls can also block X-rays. Using an approach similar to Equation 6, the coordinates of the four corners of the sidewall can be used to define the sidewall plane. Each X-ray passing through this plane can then be readily tested to determine if it is located within the sidewall region or not. If so, the $\mathrm{X}$-ray can then be rejected. 
Utilizing this algorithm for all X-rays that travel to the detectors, the model rejects all possible X-rays that are blocked by the complex shape of the holder frame. The acceptance or rejection is then included in the effective collection solid angle by,

$$
B_{i, \text { frame }}= \begin{cases}1 & \text { accept } \\ 0 & \text { reject }\end{cases}
$$

The above procedure is visualized in Supplement Movie 2. Furthermore, the X-ray acceptance and rejection conditions are illustrated in Figure 4.

In addition to the holder frame, it is found that two legs of the Softlock securing clip (50 $\mu \mathrm{m}$ thick) can block X-rays on their way to detector 2 or 3 as shown in Figure 3 The blocking effect from these clip legs can be modeled in a similar way as that for holder frame sidewalls with Eqn. 7, but with clip shadowing expressed as $B_{i, \text { clip }}$. When placed on the top of specimen, the support grid can also block low angle X-rays. As in Figure 2a, a $1 \times 2 \mathrm{~mm}$ slot-shaped hole is seen in the grid center. Considering the simple and symmetric shape, the grid geometry is modeled analytically. For each X-ray, the intercept point at the grid horizontal plane is first calculated. This enables the position comparison between the intercept point and the hole edge to assess X-ray blocking. The shadowing effect of specimen grid is then expressed in the variable $B_{i, \text { grid }}$.

\subsection{X-ray filtering by the specimen carrier}

The Be specimen carrier absorbs low energy X-rays of less than 2-3 keV but allows higher energy X-rays to penetrate through. An example can be drawn from the $\mathrm{Ni}_{3} \mathrm{Al}$ specimen where the attenuation coefficient of $\mu_{\mathrm{Al}-\mathrm{K}}$ and $\mu_{\mathrm{Ni}-\mathrm{K}}$ in $\mathrm{Be}$ is 188.8 and $1.0 \mathrm{~cm}^{-1}$, respectively[26]. In that case, $97 \%$ of $\mathrm{Al}-\mathrm{K}$ and $2 \%$ of Ni-K X-rays will be absorbed when traveling a distance of $100 \mu m$ through the Be carrier.

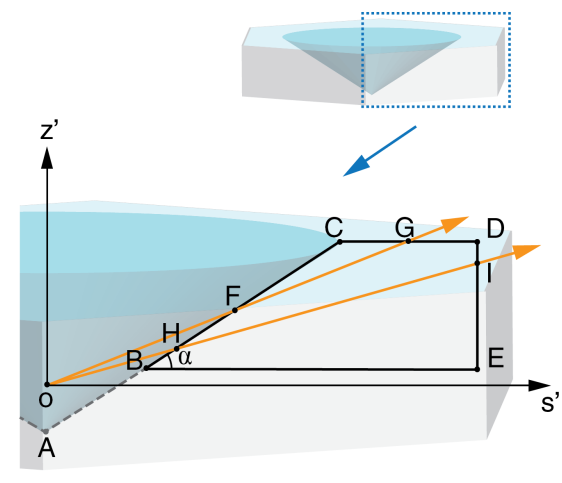

Figure 5: The geometry of the specimen carrier is treated as a hexagonal prism whose interior truncated by a cone. X-rays can travel through the Be specimen carrier via its top or its outer sidewall (i.e. path FG or path HI), resulting in X-ray filtering. 
We approximate the FEI specimen carrier as a hexagonal prism whose interior is defined by a truncated cone as shown in Figure 5. The following attenuation function is then used to calculate the penetration percentage, $A_{i, \text { carr }}$, of X-rays through the Be specimen carrier,

$$
A_{i, \operatorname{carr}}=\exp \left(-l_{i} \mu \rho_{B e}\right)
$$

where $l_{i}$ is the distance that each X-ray travels within the Be carrier, $\mu$ is the attenuation coefficient in Be carrier with its value dependent upon the incoming X-ray energy, and $\rho_{\mathrm{Be}}$ is the density of Be $(1.848$ $\left.\mathrm{g} / \mathrm{cm}^{3}\right)$.

We calculate the distance $l_{i}$ between two intercept points that each X-ray travels in and out the specimen carrier, i.e. F and G, as indicated in Figure 5. The X-ray can either exit the hexagonal prism through the top, $F_{\mathrm{I}} G_{\mathrm{I}}$, or outer sidewall, $F_{\mathrm{II}} G_{\mathrm{II}}$. The coordinates of $G$ can be analytically calculated via Equation 6 , with a slight modification, taking the plane normal $\mathbf{n}$ as $[0,0,1]$ with a rotation matrix $\mathbf{R}$,

$$
\mathbf{R}=\left(\begin{array}{ccc}
\cos \beta & 0 & \sin \beta \\
0 & 1 & 0 \\
-\sin \beta & 0 & \cos \beta
\end{array}\right)\left(\begin{array}{ccc}
1 & 0 & 0 \\
0 & \cos \alpha & -\sin \alpha \\
0 & \sin \alpha & \cos \alpha
\end{array}\right)
$$

with $\alpha$ and $\beta$ define the specimen tilt along the $\mathrm{X}$ and $\mathrm{Y}$ axes of the specimen holder respectively. The coordinates of $F\left(x_{F}, y_{F}, z_{F}\right)$ are then obtained by solving the equation of the cone surface and the linear equation of each X-ray

$$
\left\{\begin{array}{l}
\left(x-T_{x}\right)^{2}+\left(y-T_{y}\right)^{2}=\left(z+T_{x}-\frac{D}{2 \tan \lambda}\right)^{2} \cdot \tan ^{2} \lambda \\
\frac{x}{x_{i 0}}=\frac{y}{y_{i 0}}=\frac{z}{z_{i 0}}
\end{array}\right.
$$

where $\left[x_{i 0}, y_{i 0}, z_{i 0}\right]=[\sin \theta \cos \varphi, \sin \theta \sin \varphi, \cos \theta] \mathbf{R}^{-1}$ and $\lambda$ is the cone angle $\left(\sim 70^{\circ}\right) . \quad D$ is the cone open diameter of about $3.1 \mathrm{~mm}$.

By solving for $\mathrm{x}, \mathrm{y}$, and $\mathrm{z}$ from Equation 10, the coordinates $x_{F}, y_{F}, z_{F}$ of $F$ are obtained with $\left[x_{F}, y_{F}, z_{F}\right]$ $=[x, y, z] \mathbf{R}$. The distance between $F$ and $G$ are then completely defined. The absorbed X-ray fraction $A_{i, \text { carr }}$ is incorporated via Equation 3. Furthermore, it should be noted in Figures 2a that the specimen carrier has a notch that decreases the penetration distance for particular X-rays. With the precise geometry of the specimen carrier numerically defined, this subtle feature is also included in the model.

\subsection{Specimen absorption}

X-rays can also be absorbed as they travel through the specimen before reaching vacuum. Assuming that the X-rays are generated with equal probability along the beam direction, and that the path can be divided into $\mathrm{N}$ slices through the specimen thickness, the attenuation factor can be calculated for each each slice at depth of $t$ according to, 


$$
A_{i, \mathrm{spec}}=\exp \left(-s_{i, t} \mu \rho\right)
$$

where $s_{i, t}$ is the distance that each X-ray travels inside the specimen when generated at depth $t, \mu$ is the attenuation coefficient, $\rho$ is specimen density. The specimen geometry is simplified by considering that the top surface of the specimen is flat, but potentially inclined relative to the beam. This simplifying assumption is a good approximation for a sample without strong local bending or distortion. In addition to wedge-polished samples, this approximation is applicable to electropolished samples. Additional considerations, however, are required for dispersions/replicas on carbon supports due to their more complicated geometry.

For wedge shaped samples, the top surface allows two degrees of freedom regarding its angular relationship to the holder horizontal plane, i.e. inclined by $\alpha_{s}$ and $\beta_{s}$ relative to the $\mathrm{X}$ and $\mathrm{Y}$ axes of the holder. While the wedge angle can, in principle, be measured, the inclination angle is difficult to externally measure as it changes with grid mounting and local distortion specifically at thin areas. Therefore, $\alpha_{s}$ and $\beta_{s}$ are regarded as internally calibrated values that can be estimated from a tilt series prior to EDS quantification within a particular region. In other words, $\alpha_{s}$ and $\beta_{s}$ can be estimated via searching the smallest deviation between calculation and experimental results from both counts and counts ratios as a function of tilt. The search should be within the reasonable range that represents the wedge shape sample. These two angles are added as input parameters to the model in order to accurately calculate the distance $s_{i}$, after calibrating them from experimental data,

$$
s_{i, t}=\frac{\mathbf{n}_{s} \cdot[0,0, t]}{\mathbf{n}_{s} \cdot\left[\sin \theta_{i} \cos \varphi_{i}, \sin \theta_{i} \sin \varphi_{i}, \cos \theta_{i}\right]}
$$

where $\mathbf{n}_{s}$ is the plane normal of the specimen top surface, $\mathbf{n}_{s}=\mathbf{R}_{s} \mathbf{R}$ where,

$$
\mathbf{R}_{s}=\left(\begin{array}{ccc}
\cos \beta_{s} & 0 & \sin \beta_{s} \\
0 & 1 & 0 \\
-\sin \beta_{s} & 0 & \cos \beta_{s}
\end{array}\right)\left(\begin{array}{ccc}
1 & 0 & 0 \\
0 & \cos \alpha_{s} & -\sin \alpha_{s} \\
0 & \sin \alpha_{s} & \cos \alpha_{s}
\end{array}\right)
$$

Also, the bottom plane of the specimen can generally be ignored as the detected X-rays only escape from the specimen top surface when using the FEI low background double tilt specimen holder. For tomography holder, however, X-rays can exit from the bottom plane of holder when orientated at high angles of tilt. Note that when using a single EDS detector, the absorption correction for an inclined surface of a wedge-shaped sample was previously developed in Ref. [27].

\section{Model Validation and Insights}

To validate the model, we compare experiment and calculations of X-ray signals collected as a function of specimen tilt using a wedge-shaped $\mathrm{Ni}_{3} \mathrm{Al}$ specimen. $\mathrm{Ni}_{3} \mathrm{Al}$ is chosen as an excellent test candidate due to 
its well separated $\mathrm{Ni}$ and $\mathrm{Al}$ characteristic X-ray peaks and the strong absorption of Al-K X-ray by Ni. The specimen has inclination angles of $1^{\circ}$ and $3^{\circ}\left(\alpha_{s}\right.$ and $\beta_{s}$ ) relative to $\mathrm{X}$ and $\mathrm{Y}$ axis of the holder, respectively, as determined by comparing predicted absolute counts and signal ratios to experiment.

With regards to absolute counts, Figures 6 shows that the experimental Al-K and Ni-K counts change in a complicated fashion as a function of X-tilt from $-30^{\circ}$ to $+30^{\circ}$. The effective solid angle and precise tilt conditions are predicted from the model with all the contributions described above. Furthermore, the predicted X-ray intensity is calculated via Equation 4, which allows absolute comparison to experiment.

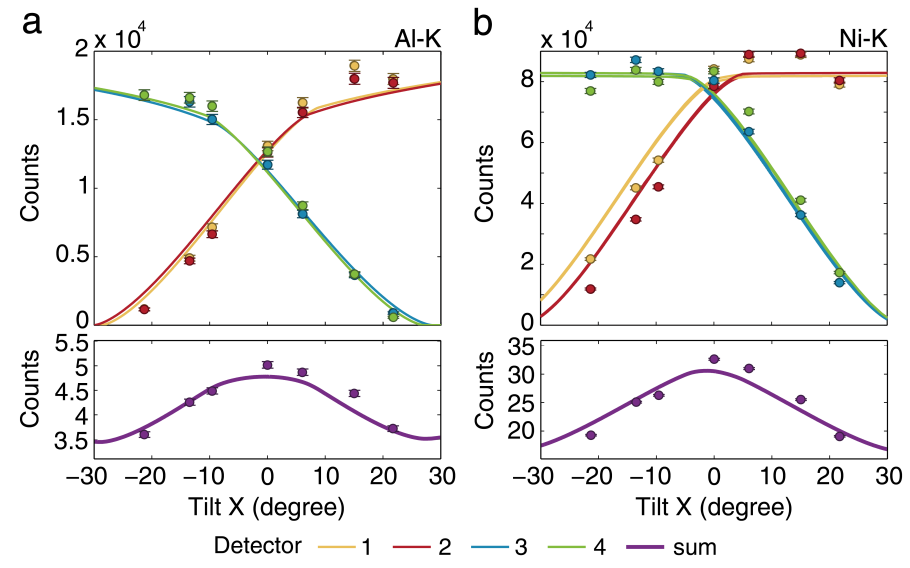

Figure 6: Experiment (dots) and prediction (solid lines) of (a) Al-K and (b) Ni-K counts as a function of X-tilt from $-30^{\circ}$ to $+30^{\circ}$. Colors are used to indicate the individual detectors and their sum.

As shown in Figure 6, the calculated (solid lines) and experimental results (dots) show excellent agreement for each individual detector as well as the detector sum. For example, consider detector 2. Al-K counts gradually increase during $-30^{\circ}$ to $5^{\circ}$ of tilt, followed by a slow increase as shown in Figure 6a. Similarly, the Ni-K counts gradually increase from $-30^{\circ}$ to $5^{\circ}$ of tilt in Figure $6 \mathrm{~b}$. As tilt increases, the Ni-K counts remain approximately constant compared to the Al-K, indicating less specimen absorption.

From insights provided by the numerical model, the behavior of $\mathrm{Ni}-\mathrm{K}$ and $\mathrm{Al}-\mathrm{K}$ signals in the $-30^{\circ}$ to $5^{\circ}$ range results from the combined effects from shadowing and carrier filtering. The gradual increase for Al-K for the positive tilt range results from strong specimen absorption. Similar trends are also found in Figure 7 when the specimen is tilted along $\mathrm{Y}$-axis. In contrast to the $\mathrm{X}$-axis tilt, Ni-K counts from the detector pair $2 / 3$ reach a lower maximum than that from the detector $1 / 4$ pair due to the additional shadowing effects from Softlock securing clip.

When the signals from all four detectors are summed, the maximum Al-K and Ni-K counts are observed near zero tilt, indicating that the combined effects are optimized for all detectors simultaneously. This confirms that zero or near-zero specimen tilt is the preferred experimental condition to obtain the highest Xray acquisition rate[4]. In many situations, this may not be practical due to other constraints, e.g. achieving 
zone axis conditions for atomic resolution elemental mapping. Furthermore, we note that some experimental counts appear to be higher than the model predictions. While strong channeling conditions were avoided, some tilt conditions may be closer to channeling conditions than others, which is not accounted for in the model.

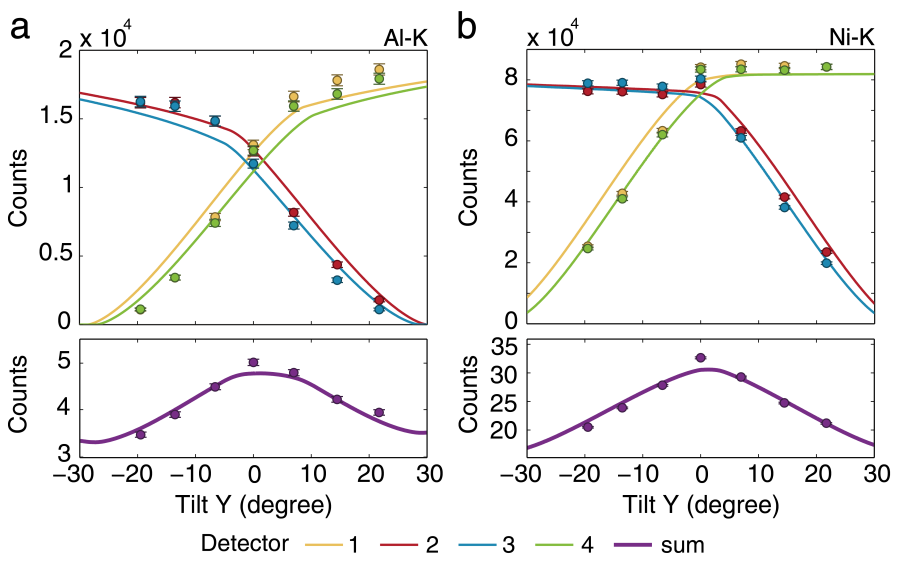

Figure 7: Experiment (dots) and prediction (solid lines) of (a) Al-K and (b) Ni-K counts as a function of Y-tilt from -30 ${ }^{\circ}$ to $+30^{\circ}$. Colors are used to indicate the individual detectors and their sum.

In situations where one X-ray type may be preferentially absorbed within the specimen, e.g. Al-K in $\mathrm{Ni}_{3} \mathrm{Al}$, the intensity ratio, $\mathrm{Al}-\mathrm{K} / \mathrm{Ni}-\mathrm{K}$, can be strongly influenced by the four quadrant geometry as in Figure 8. Despite the similarity in their general trends, the intensity ratio for each detector is quite distinct. As in Figure 8a for example, the intensity ratio from detector 1 is higher than that from detector 2 at positive tilt angles, while the trend is reversed at negative tilts. A similar trend is also seen from detector pair 3 and 4. Furthermore, the separation of the intensity ratio for detectors 1 and 2 is larger than that between detectors 3 and 4 .

When all detectors signals are summed, the intensity ratio is found to vary with respect to tilt angle. While the ratio deviates slowly for small tilt angles, the deviation introduced at high tilt angles can be as large as 30\%. Critically, the presented model quantitatively reproduces the observed trends. Moreover, this result demonstrates the need for calculated absorption correction factors based on specimen tilt conditions to conduct quantitative EDS analysis with the Super-X detector system.

Using either the absolute counts or intensity ratio, the estimated composition can be obtained from Equation 4 or 5. Comparing with the nominal composition of $\mathrm{Ni}_{3} \mathrm{Al}$ (Al:13.3 wt.\%, Ni:86.7 wt.\%), the relative error from model calculation is reported in Figure 9. The composition errors from the intensity ratio show systematically smaller variation than that from single counts. In most cases, Al-K and Ni-K counts show deviation in the same direction. While individual detectors show relatively large errors, such variance is 'averaged out' by summing the contribution from all detectors. Particularly for higher tilts, 
a

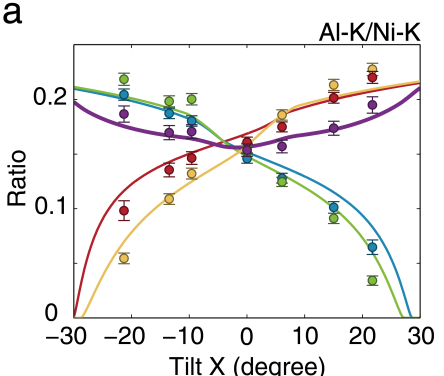

Detector $-1-2-3-4-$ sum

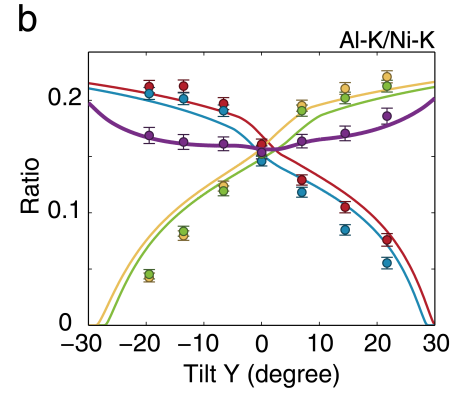

Figure 8: Experiment (dots) and model predictions (solid lines) of of Al-K/Ni-K intensity ratios as a function of (a) X- and (b) Y-tilt from $-30^{\circ}$ to $+30^{\circ}$. Colors are used to indicate the intensity ratio for each detector and the detector sum..

however, the relative error can reach $\sim 10 \%$ for the detector sum. These above findings suggest a counterbalancing effect from Super-X four-quadrant configuration, but that a more symmetric holder geometry along the X-ray take-off direction from specimen to each detector would benefit quantification. Based on our calculations, we find that there is a discrepancy in beam blocking to the different detectors when using the FEI low background holder. In particular, the larger sidewall height along the X-ray path to detectors $2 / 3$ introduces additional blocking as compared to the path to detectors $1 / 4$. The securing clip legs cause further X-ray blocking along the path detectors $2 / 3$. Therefore, reducing the sidewall height or redesigning the position of securing clip is suggested for optimum holder geometry.

\subsection{Individual contributions to the effective solid angle}

To better describe the contributions to the calculated X-ray counts, two-dimensional maps of the detector collection can be calculated as a function of specimen tilt, see Figure 10. Generally, the maximum counts are found at one corner of the map, while at the opposing corner zero counts are collected. This directly corresponds to the four-quadrant Super-X detector geometry, as the detectors are positioned symmetrically with respect to the $\mathrm{X}$ and $\mathrm{Y}$-axes. Further, the maps also exhibit a flat and non-symmetrical, ' $\mathrm{V}$ ' shaped contour.

Regarding the intensity ratio maps, Figures 10i-l, a maximum ratio is also seen at one map corner. The ' $V$ ' shape from the counts map is not directly reflected on the ratio map, but the abrupt change of the intensity ratio trend occurs at a tilt of $\pm 15-30^{\circ}$ along X-tilt and $\pm 5^{\circ}$ along $\mathrm{Y}$-axis . Beyond the similar trends, slight differences in the contour positioning and spacing occurs between the individual detectors. This is due to the combined effects of holder geometry, specimen shift and/or specimen geometry.

The benefit of the numerical model presented here is that each contribution to Equation 3 can be investigated separately. In particular, the (i) specimen absorption, (ii) specimen carrier filtering, (iii) clip shadowing, (iv) holder frame shadowing, and (v) grid shadowing are sequentially added and compared in Figure 11. The general shape of the contours is dominated by specimen absorption as shown in Figures 

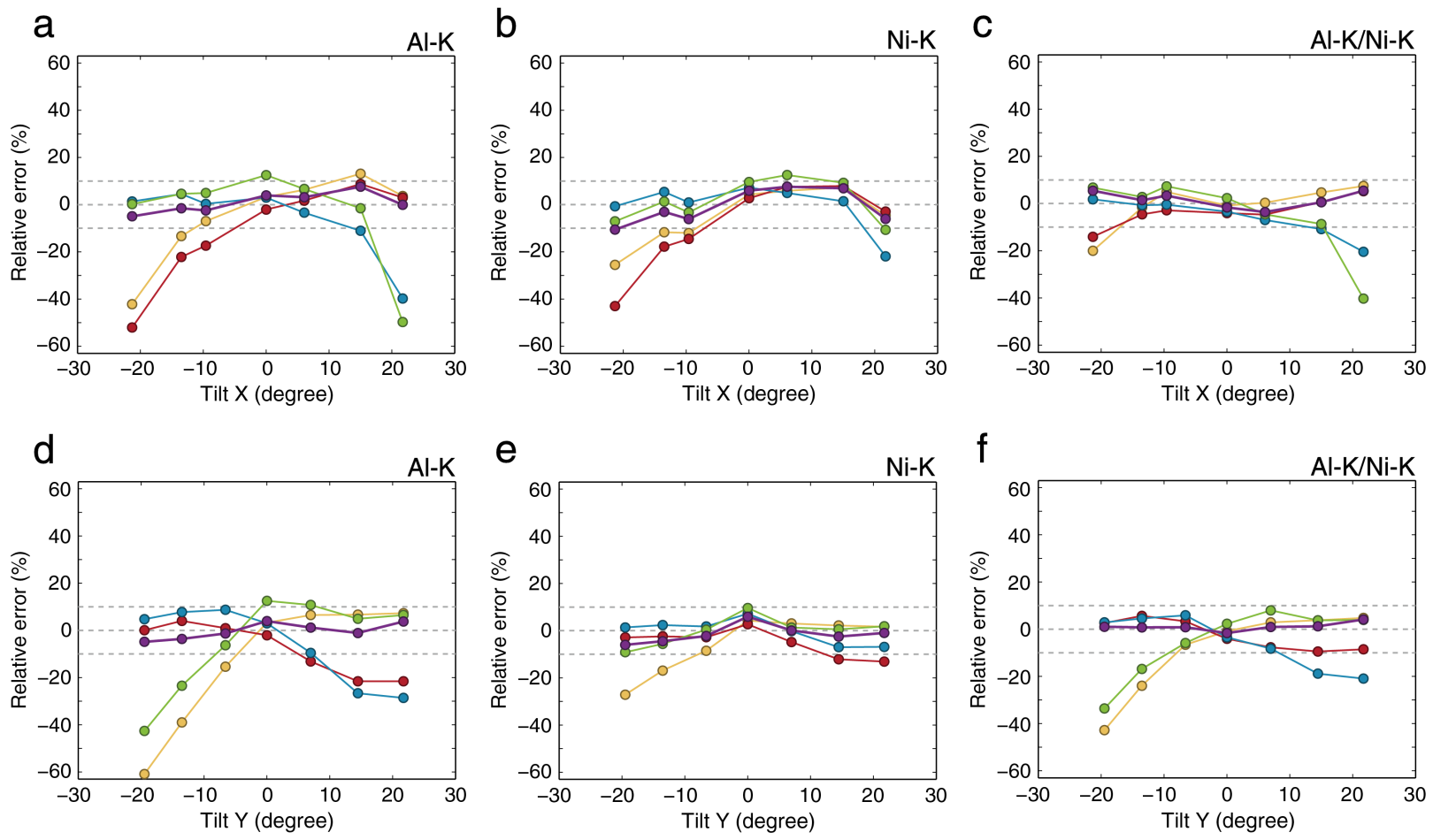

Detector $-1-2-3-4-$ sum

Figure 9: Relative error of the predicted composition from (a) Al-K counts, (b) Ni-K counts, (c) Al-K/Ni-K intensity ratio as a function of X-tilt. Relative error of the predicted composition from (d) Al-K counts, (e) Ni-K counts, (f) Al-K/Ni-K intensity ratio as a function of Y-tilt. Dashed lines mark $\pm 10 \%$ error.

11a, f and k. Furthermore, stronger specimen absorption occurs for Al-K than for Ni-K as expected. With the Be carrier filtering added, a significant drop of Al-counts is seen in Figure 11b. Ni-K counts are also slightly reduced in Figure 11g, indicating the smaller absorption of Ni-K X-rays by Be. In this way, the filtering effect causes a decrease of the intensity ratio, as shown in Figure 11l. Furthermore, the Softlock clip significantly shadows Ni-K X-ray, which can be seen from the contour shifts of Figure 11h.

The ' $\mathrm{V}$ ' shaped contours originate from X-rays that are blocked by the holder frame. As seen in Figure 11i, strong holder shadowing occurred at negative $\mathrm{X}$ and $\mathrm{Y}$ tilt directions. The increase of $\mathrm{Al}-\mathrm{K} / \mathrm{Ni}-\mathrm{K}$ can be seen in Figure 11n when holder shadowing is present. In fact, specimen absorption and holder carrier filtering will cause a smaller $\mathrm{Al}-\mathrm{K} / \mathrm{Ni}-\mathrm{K}$ intensity at lower X-ray takeoff angles. It is the blocking of these low takeoff angle X-rays that eventually increases the overall intensity ratios. Consequently, the Al-K/Ni-K intensity ratio is found to be counter balanced by the specimen absorption/filtering effects and shadowing effects, i.e. the former decreases the ratio and the latter increase the ratio. The grid shadowing has little impact as shown Figure 11j, with only slight shifts in the contour lines.

With all the detector signals summed, the overall Al-K and Ni-K counts are given in Figure 12a, b. The 


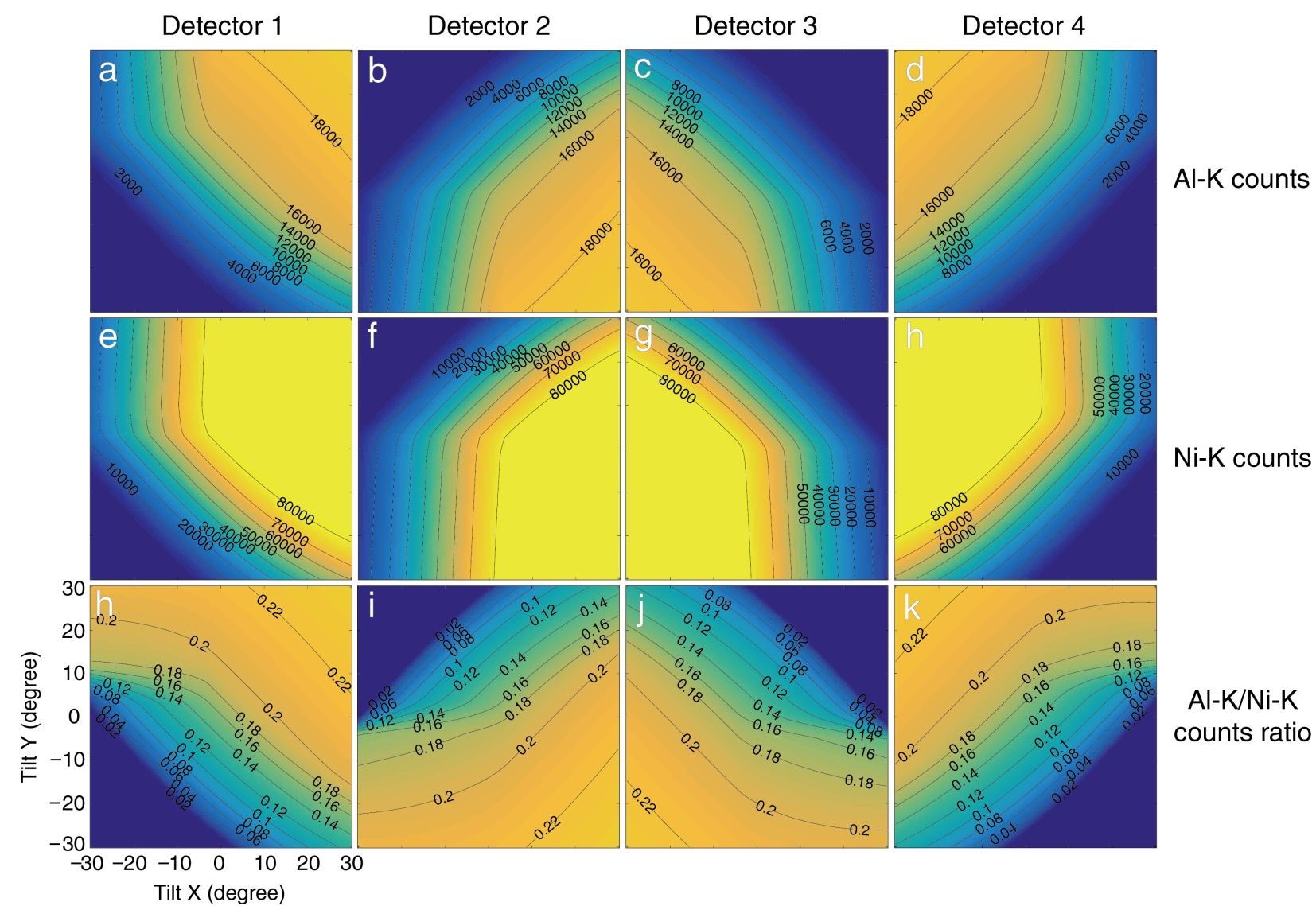

Figure 10: Two dimensional response map of each individual detector to Al-K counts, Ni-K counts, and Al-K/Ni-K intensity ratio along both X- and Y-tilts.

peak of the map appears to be located near the zero tilt position. However, significant variation of the Al-K and Ni-K counts occurs with respect to tilt. This indicates that composition quantification based on the absolute X-ray counts can be very sensitive to specimen tilt. In contrast, the variation of the intensity ratio map is much smaller. As shown in Figure 12c, the change of ratio within $\pm 15^{\circ}$ of $\mathrm{X}$ or $\mathrm{Y}$ tilt varies about 5\%. In this region, it may still be reasonable to apply a constant k-ratio factor for EDS analysis. At higher tilts, the variation of the ratio also becomes larger, indicating a correction factor should to be used under these conditions.

\subsection{Influence of specimen shift}

Moving the sample within the pole-piece gap changes the location of X-rays emanating from the specimen. This alters the geometric relationship between the X-rays and the holder. Figure 13 shows maps of Al-K and $\mathrm{Ni}-\mathrm{K}$ counts and ratio in the range of a $\pm 0.5 \mathrm{~mm}$ shift of the specimen in $\mathrm{X}$ and $\mathrm{Y}$ direction.

At zero tilt, both the absolute counts and intensity ratio show minimal variance within $\pm 0.5 \mathrm{~mm} \mathrm{X}$-shift and $\pm 0.2 \mathrm{~mm}$ Y-shift. A significant drop occurs, however, beyond $\pm 0.3 \mathrm{~mm}$ of $\mathrm{Y}$-shift. This is due to the 


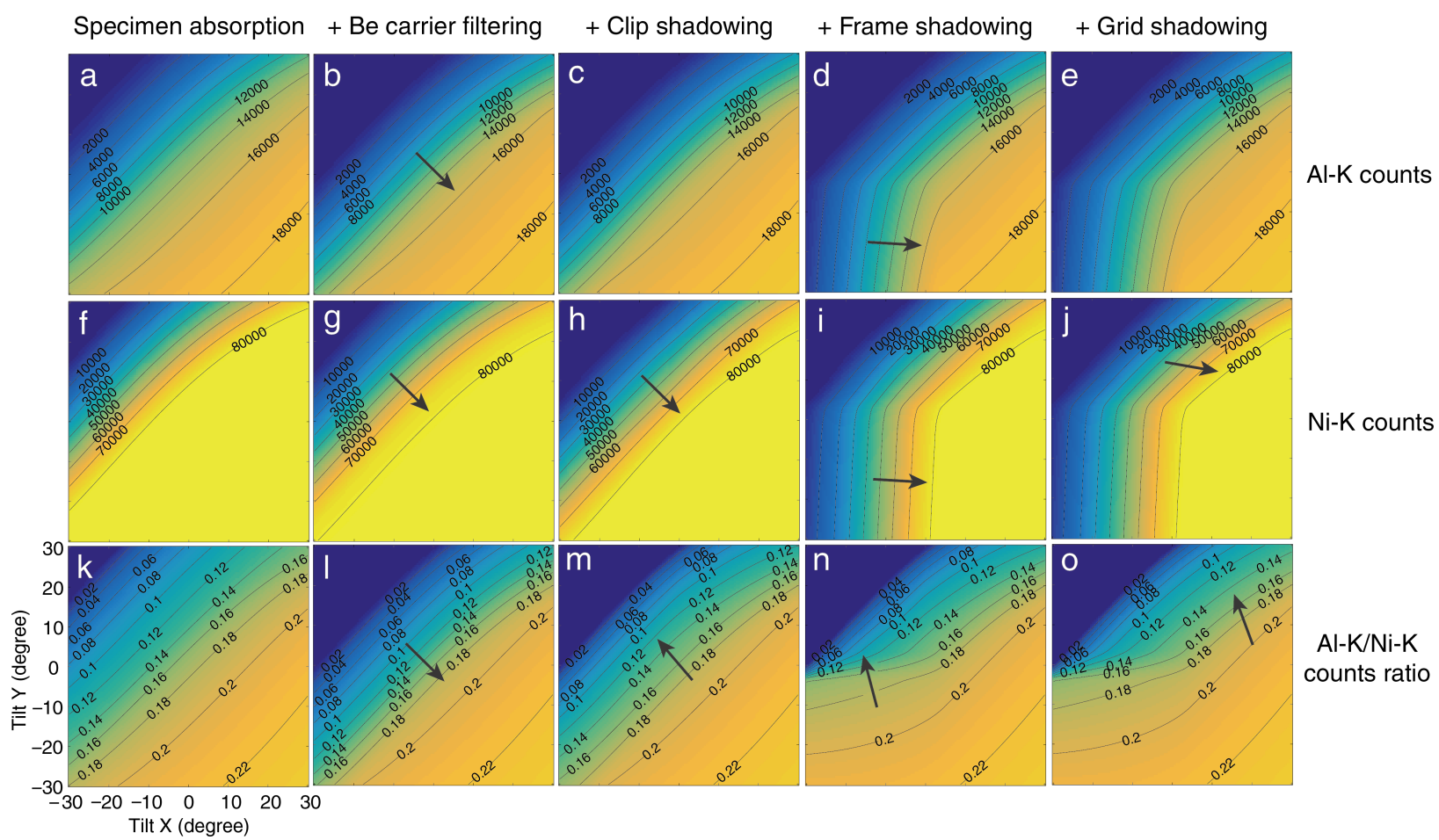

Figure 11: Two dimensional response map of Al-K counts, Ni-K counts, and the Al-K/Ni-K intensity ratio along both X- and Y-tilt are shown for detector 2. Arrows are added to show the contour shift.
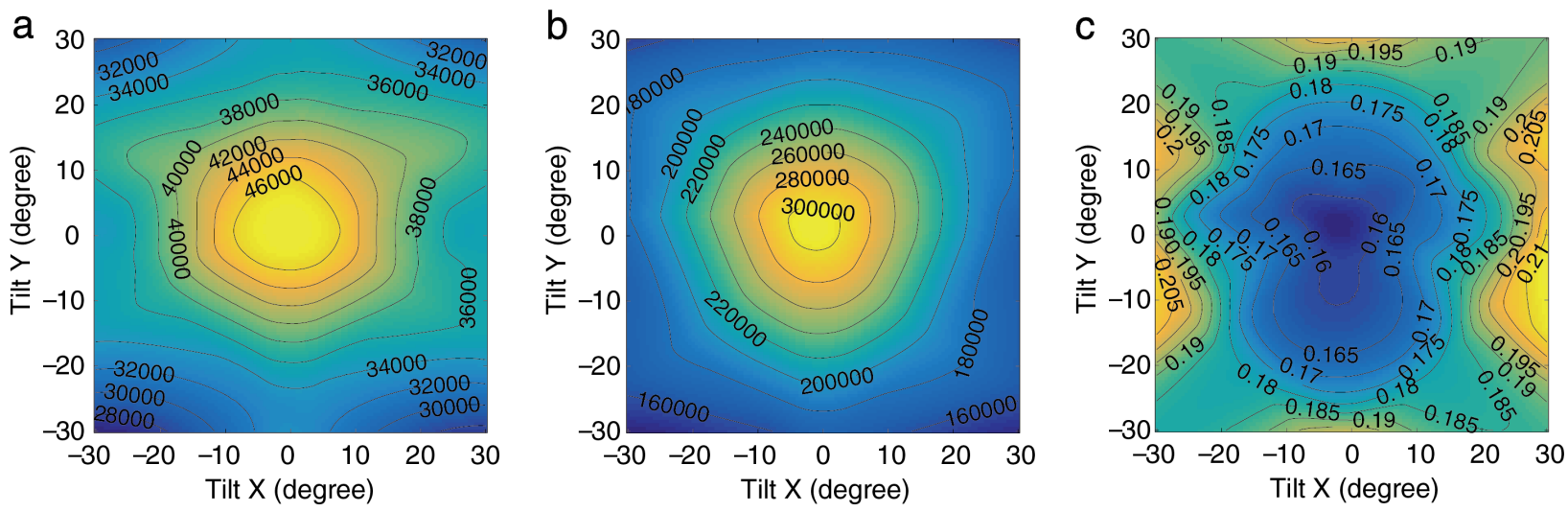

Figure 12: Two dimensional response map of overall (a) Al-K counts, (b)Ni-K counts, and (c) the Al-K/Ni-K intensity ratio along both $\mathrm{X}$ and Y-tilt for detector sum.

strong shadowing effect as the probed region of the specimen approaches the grid edge. A similar response is obtained when the specimen is tilted with 20 degrees in X and Y, as seen in Figure 13d-f. Considering that $\mathrm{X}$ and $\mathrm{Y}$ shifts are typically within $\pm 0.2 \mathrm{~mm}$ under most experimental conditions, grid shadowing will generally not play an important role. The influence of specimen shift can thus be regarded as a fairly minor perturbation on the collected X-rays. 

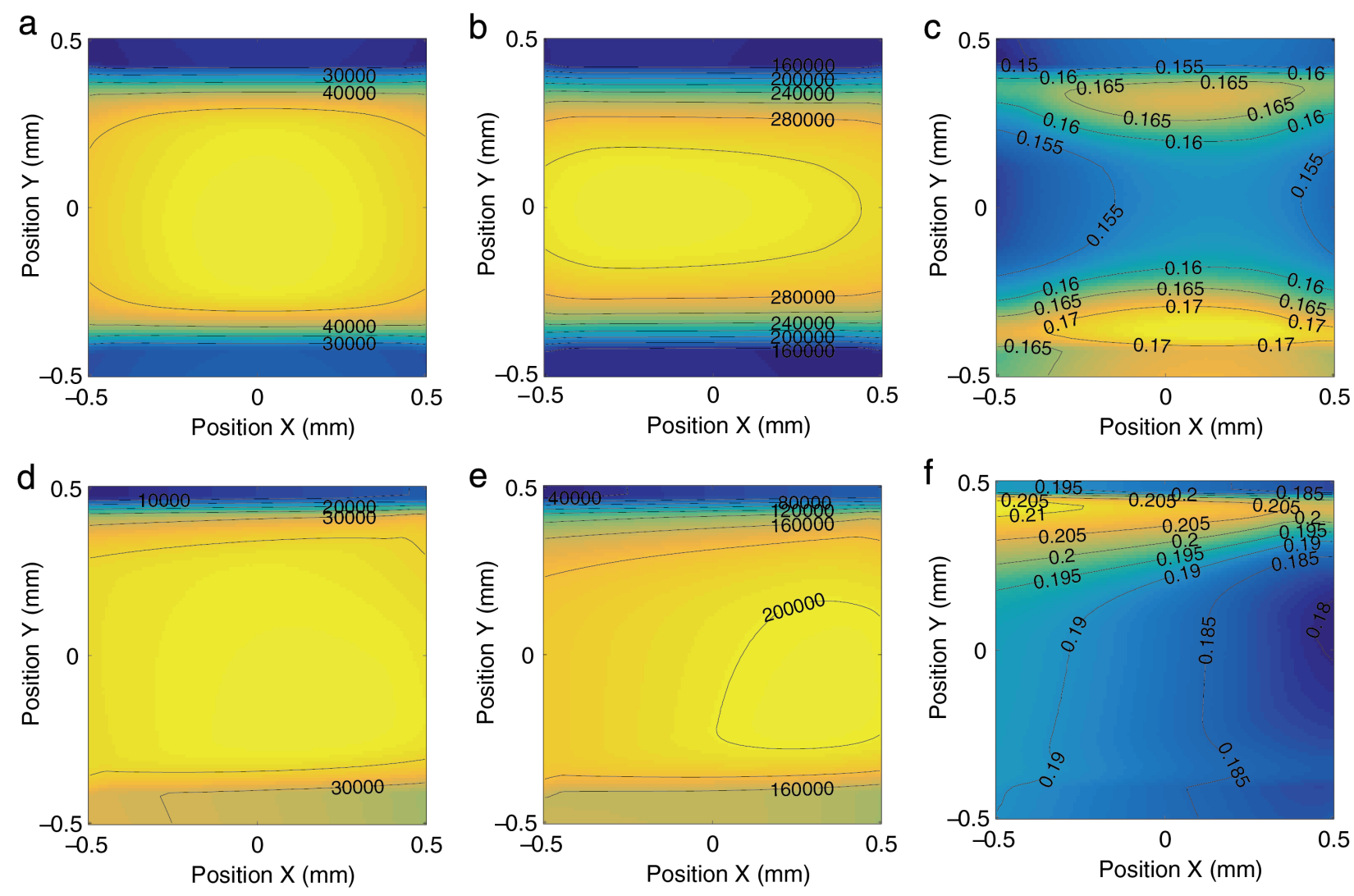

Figure 13: Two dimensional response map for (a) Al-K (b) Ni-K counts and (c) Al-K/Ni-K intensity ratio for the specimen shifted in the $\mathrm{x}$ and $\mathrm{y}$ directions. (d) Al-K (e) Ni-K counts and (f) the Al-K/Ni-K intensity ratio with the specimen tilted $+20^{\circ}$ in $\mathrm{X}$ and $\mathrm{Y}$ and shifted in $\mathrm{x}$ and $\mathrm{y}$ directions.

\section{Spurious X-ray generation}

To examine the effect of spurious X-rays, a FIB cut $\mathrm{Ni}_{3} \mathrm{Al}$ specimen is considered. For the model, the FIB specimen surface is approximately flat. With the the FIB specimen attached to a ' $M$ '-shaped Mo grid close to the grid sidewall, the Al-K counts measured in the experiment are found to deviate significantly from the model as shown in Figure 14a. Likewise, the Ni-K counts for detector 1 and 2 are also higher than the model prediction as in Figure 14b. Concomitantly, a sharp rise in the Mo-K and Mo-L counts is observed in the same spectrum as shown in Figures $14 \mathrm{c}$ and d.

Regarding the source of the spurious signal, a large Mo K/L ratio indicates that stray X-rays are likely the cause, while a low Mo K/L ratio signals stray electrons[1]. The low Mo K/L ratio from detector 3 and 4 can thus be attributed to stray electrons, where the scattered electrons happen to strike the grid sidewall. For detectors 1 and 2, the comparatively larger Mo K/L ratio indicates the presence of stray X-rays. The strong Mo-K/L X-rays, as well as the Bremsstrahlung background, can flood the grid and specimen. As a result, $\mathrm{Al}-\mathrm{K}$ and $\mathrm{Ni}-\mathrm{K}$ characteristic X-rays fluoresced from a large area, thereby increasing the absolute 

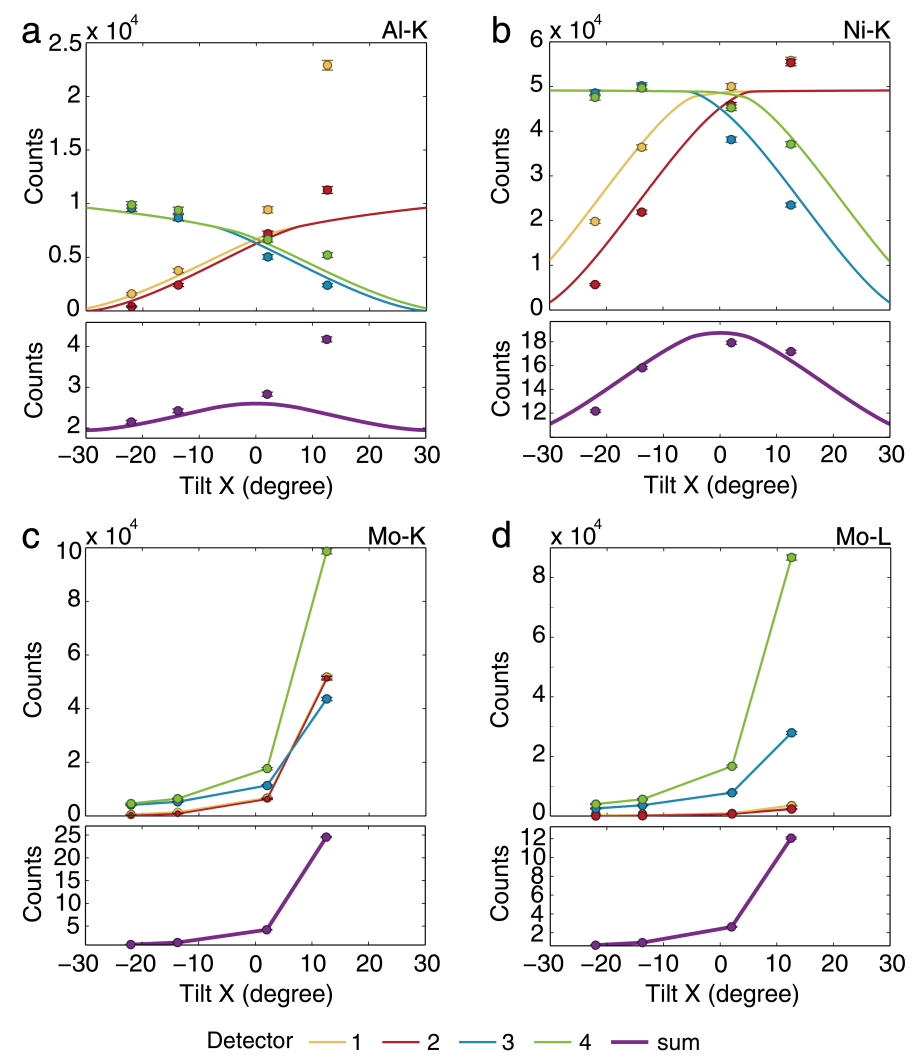

Figure 14: Predicted (solid lines) and experiment (dots) (a) Al-K and (b) Ni-K counts, (c) Mo-K and (d) Mo-L counts as a function of tilt from $-30^{\circ}$ to $+30^{\circ}$ X-tilt for the FIB prepared sample. Colors indicate each individual detector or the detector sum.

counts beyond the predicted model.

The increment of Al-K or Ni-K counts in Figure 14a and b, however, cannot be directly linked to the change int Mo signal. Instead, other sources are likely present. For example, backscattered electrons or secondary electrons can also play a role in generating the spurious X-ray from the sample. Given the complexities associated with spurious X-rays, caution should be taken for sample preparation geometries prone to their generation.

\section{Conclusion}

A numerical model has been established that incorporates the detailed holder geometry and the combined effects of (i) specimen absorption, (ii) specimen carrier filtering, (iii) clip shadowing, (iv) holder frame shadowing, and (v) grid shadowing. The numerical predictions of Al-K and Ni-K counts derived from the model have been shown to be in excellent agreement with experiment as a function of $\mathrm{X}$ and $\mathrm{Y}$ tilt from $-30^{\circ}$ to $+30^{\circ}$. Furthermore, the qualitative and quantitative agreement with experiment across the tilt range enables the capability to calculate correction factors for improved quantification accuracy regardless 
of tilt. While the individual detectors show strong variation in effective collection efficiency with tilt, the detector sum largely shows a balanced total with reduced error. As a next step, more complicated specimen geometries can be readily included in the numerical model to investigate its role on influencing energy dispersive $\mathrm{x}$-ray spectroscopy with multiple detectors.

\section{Appendix A. Supporting Information}

The following describes the Supplementary movies related to this article.

Movie 1. An illustration of the specimen holder shadowing as it is tilted along X and Y directions.

Movie 2. Visualization of the algorithm that is used to determine if X-rays are blocked by the specimen holder.

\section{Acknowledgements}

The authors gratefully acknowledge the Air Force Office of Scientific Research (FA9550-14-1-0182) for support of this research. Adedapo Oni is thanked for preparing the specimens. The authors also acknowledge the Analytical Instrumentation Facility (AIF) at North Carolina State University, which is supported by the State of North Carolina and the National Science Foundation.

[1] D. William, C. Carter, Transmission Electron Microscopy, Second Edition, Springer, New York, 2009.

[2] N. J. Zaluzec, Innovative Instrumentation for Analysis of Nanoparticles: The $\pi$ Steradian Detector, Microscopy Today 17 (2009) 56-59

[3] P. Kotula, P. Lu, D. Susan, Z. Ghanbari, J. Ringnalda, Progress with the Sandia Titan G2 80-200 with 0.7sr Integral SDD Array, Microscopy and Microanalysis 18 (2012) 324-325.

[4] P. Schlossmacher, D. Klenov, B. Freitag, S. Harrach, A. Steinbach, Nanoscale Chemical Compositional Analysis with an Innovative S/TEM-EDX System, Microscopy and Analysis 24 (2010) 55.

[5] P. Schlossmacher, D. Klenov, B. Freitag, H. von Harrach, Enhanced Detection Sensitivity with a New Windowless XEDS System for AEM Based on Silicon Drift Detector Technology, Microscopy Today 18 (2010) 14-20.

[6] S. Isakozawa, K. Kaji, K. Tamura, X. Zhang, A. Sandborg, N. Baba, The development of a new windowless XEDS detector, Journal of Electron Microscopy 59 (6) (2010) 469-472.

[7] A. J. D'Alfonso, B. Freitag, D. Klenov, L. J. Allen, Atomic-resolution chemical mapping using energy-dispersive x-ray spectroscopy, Phys. Rev. B 81 (2010) 100101.

[8] B. D. Forbes, A. J. D'Alfonso, R. E. A. Williams, R. Srinivasan, H. L. Fraser, D. W. McComb, B. Freitag, D. O. Klenov, L. J. Allen, Contribution of thermally scattered electrons to atomic resolution elemental maps, Phys. Rev. B 86 (2012) 024108 .

[9] M. Watanabe, Microscopy Hacks: development of various techniques to assist quantitative nanoanalysis and advanced electron microscopy, Microscopy 62 (2) (2013) 217-241.

[10] G. Kothleitner, M. J. Neish, N. R. Lugg, S. D. Findlay, W. Grogger, F. Hofer, L. J. Allen, Quantitative Elemental Mapping at Atomic Resolution Using X-Ray Spectroscopy, Phys. Rev. Lett. 112 (2014) 085501.

[11] P. Lu, L. Zhou, M. Kramer, D. J. Smith, Atomic-scale Chemical Imaging and Quantification of Metallic Alloy Structures by Energy-Dispersive X-ray Spectroscopy, Scientific Reports 4 (2014) 3945. 
[12] P. Lu, M. Van Benthem, Q. Jia, Chemical Quantification of Atomic-scale EDS Maps under Thin Specimen Conditions, Microscopy and Microanalysis 19 (2013) 1298-1299.

[13] Z. Chen, A. D’Alfonso, M. Weyland, D. Taplin, L. Allen, S. Findlay, Energy dispersive x-ray analysis on an absolute scale in scanning transmission electron microscopy, Ultramicroscopy 157 (2015) 21-26.

[14] T. Slater, P. Camargo, M. Burke, N. Zaluzec, S. Haigh, Understanding the limitations of the super-x energy dispersive $\mathrm{x}$-ray spectrometer as a function of specimen tilt angle for tomographic data acquisition in the s/tem, Journal of Physics: Conference Series 522 (1) (2014) 012025.

[15] C. S. Yeoh, D. Rossouw, Z. Saghi, P. Burdet, R. K. Leary, P. A. Midgley, The Dark Side of EDX Tomography: Modeling Detector Shadowing to Aid 3D Elemental Signal Analysis, Microscopy and Microanalysis 21 (2015) 759-764.

[16] F. Yang, F. Scheltens, D. McComb, D. Williams, M. De Graef, Absorption Corrections for a Four-Quadrant SuperX EDS Detector, Microscopy and Microanalysis 20 (2014) 100-101.

[17] T. Slater, A. Janssen, P. Camargo, M. Burke, N. Zaluzec, S. Haigh, STEM-EDX tomography of bimetallic nanoparticles: A methodological investigation, Ultramicroscopy 162 (2016) 61-73.

[18] T. Malis, S. C. Cheng, R. F. Egerton, EELS log-ratio technique for specimen-thickness measurement in the TEM, Journal of Electron Microscopy Technique 8 (2) (1988) 193-200.

[19] D. Mitchell, B. Schaffer, Scripting-customised microscopy tools for digital micrograph ${ }^{\text {TM }}$, Ultramicroscopy 103 (4) (2005) $319-332$

[20] N. J. Zaluzec, Analytical Formulae for Calculation of X-Ray Detector Solid Angles in the Scanning and Scanning/Transmission Analytical Electron Microscope, Microscopy and Microanalysis 20 (2014) 1318-1326.

[21] M. Watanabe, D. B. Williams, The quantitative analysis of thin specimens: a review of progress from the Cliff-Lorimer to the new $\zeta$-factor methods, Journal of Microscopy 221 (2) (2006) 89-109.

[22] N. J. Zaluzec, Analytical Electron Microscopy, San Francisco Press, San Francisco, 1984, Ch. K- and L-shell cross sections for X-ray microanalysis in an AEM, pp. 279-284.

[23] R. Egerton, S. Cheng, Characterization of an analytical electron microscope with a nio test specimen, Ultramicroscopy 55 (1) (1994) $43-54$.

[24] W. Bambynek, B. Crasemann, R. W. Fink, H. U. Freund, H. Mark, C. D. Swift, R. E. Price, P. V. Rao, X-Ray Fluorescence Yields, Auger, and Coster-Kronig Transition Probabilities, Rev. Mod. Phys. 44 (1972) 716-813.

[25] G. Cliff, G. W. Lorimer, The quantitative analysis of thin specimens, Journal of Microscopy 103 (2) (1975) $203-207$.

[26] J. Goldstein, A. Romig Jr., C. Newbury, D.E. Lyman, F. C. Echlin, P., D. Joy, E. Lifshin, Scanning Electron Microscopy and X-Ray Microanalysis, Second Edition, Plenum Press, New York and London, 1992.

[27] N. J. Zaluzec, The Geometry of the Absorption Corrections for Analytical Electron Microscopy, Microbeam Analysis, ed. R. Geiss, San Francisco Press (1981) 325-328. 